\title{
Une approche évolutive des «visions du monde » pour penser les transformations de l'agriculture
}

\author{
Cyrille Rigolot ${ }^{*}$
}

UMR territoires, université Clermont Auvergne, AgroParisTech, Inra, Irstea, VetAgro Sup, 63000 Clermont-Ferrand, France

\begin{abstract}
Résumé - Dans les années à venir, l'agriculture sera confrontée à des défis sans précédent et devra se transformer radicalement. Des solutions très contrastées commencent à apparaître, parfois dans des directions radicalement opposées, entre l'agriculture 3.0. et la permaculture. Dans ce contexte, cet article vise à montrer l'intérêt d'une approche évolutive du concept de "visions du monde » (worldview) pour penser les transformations de l'agriculture. La première partie présente ce qu'est une vision du monde : un système structurant de signification qui informe comment les humains interprètent et co-créent la réalité. Quatre grands types de vision du monde ont été distingués dans la littérature: Traditionnelle; Moderne; Postmoderne; Intégrale. Selon une approche évolutive, ces grands types peuvent être vus comme des stades successifs dans le développement individuel et collectif, bien qu'aucun type ne puisse être considéré comme intrinsèquement "meilleur» qu'un autre. Dans la deuxième partie de l'article, je propose une déclinaison des quatre visions dans le domaine de l'agriculture. Les visions Moderne et Postmoderne sont distinguées notamment par la mise en avant des notions de sécurité alimentaire pour la première, et de souveraineté pour la seconde. La vision Intégrale, encore très peu répandue, correspond à des formes d'agriculture intégrant des dimensions scientifiques et spirituelles. Selon l'approche proposée, les transformations de l'agriculture peuvent être pensées en lien avec l'évolution dynamique des visions du monde des acteurs. Cela invite à développer une réflexivité sur sa propre vision du monde, mieux prendre en compte celle des autres, et s'engager dans des dynamiques de transformations mutuelles. De cette manière, la coexistence des systèmes peut être envisagée de façon constructive, à la fois bienveillante et engagée.
\end{abstract}

Mots clés : visions du monde / systèmes agricoles / transformations / agroécologie / coexistence

Abstract - An evolutionary approach of worldviews to think the transformations of agriculture. In the coming years, agriculture will have to face unprecedented challenges and to transform itself radically. Today already, contrasted solutions are emerging, from 3.0. agriculture to permaculture. In this context, this paper aims to show the interest of an evolutionary approach of worldviews to think the transformations of agriculture. In the first part, a worldview is defined as a structuring system of meaning, informing how humans interpret and co-create reality. Four major types of worldviews have been identified in literature: Traditional; Modern; Postmodern; Integral. Following an evolution approach, these four types can be seen as successive stages of individual and collective development, although no type can be considered as intrinsically "better" than another. In the second part of the paper, I propose an application of the four types to the agricultural domain. Modern and Postmodern worldviews put an emphasis rather on food security for the first one, and on food sovereignty for the second. The Integral worldview, still very uncommon nowadays, integrates both scientific and spiritual dimensions. The evolutionary approach enables to think differently about agricultural systems technical and organizational transformations, as coevolving with worldviews. It can also be useful to develop self-reflexivity on our own vision, better integrate those of others and engage in mutual transformations. Finally, agricultural systems coexistence can be considered in a constructive, benevolent and committed way.

Keywords: worldview / agricultural systems / transformations / agroecology / coexistence

\footnotetext{
$\overline{* \text { Auteur de correspondance }}$ : cyrille.rigolot@inra.fr
} 


\section{Introduction}

L'agriculture connaît aujourd'hui de profondes mutations, dans un contexte de grande incertitude. Selon les projections les plus plausibles, la demande mondiale en produits agricoles devrait augmenter de $70 \%$ d'ici 2050 , du fait de la croissance démographique et des changements alimentaires liés à l'augmentation des niveaux de revenu (Tilman et al., 2011). L'agriculture connaît aussi des transitions géographiques importantes, liées entre autres aux phénomènes de globalisation et d'urbanisation (Wilkinson, 2009). Dans les décennies à venir, ces transitions continueront avec des facteurs d'incertitude tels que le changement climatique, les risques sanitaires de maladies émergentes, les évolutions économiques, politiques... (Darnhofer et al., 2012). Dans le même temps, les formes actuelles d'agriculture sont aujourd'hui fortement questionnées, au Nord et au Sud (McIntyre, 2009), et de façon globale sur le plan de leur durabilité environnementale (contribution au changement climatique, à l'érosion de la biodiversité... [Tilman et al., 2011]). Dans un tel contexte, les agricultures ne pourront pas rester telles que nous les connaissons actuellement dans les années à venir : les systèmes agricoles sont appelés à se transformer radicalement vers des formes inédites.

En pratique, en effet, il est déjà possible aujourd'hui de constater l'émergence de modèles de rupture, très divers et contrastés (Conway, 1999; Hubert et al., 2013). En particulier, avec la révolution numérique, une « agriculture 3.0. » se met en place, articulant l'agriculture de précision, l'agriculture connectée et la biologie prédictive (Issac et Poyat, 2015). De façon concomitante avec cette évolution, une diversité de systèmes agroécologiques se développe, à l'échelle française comme à l'échelle mondiale (Altieri, 2009). Parmi ces formes agroécologiques nouvelles ou « revisitées », certaines comme la permaculture constituent des alternatives radicales dans nos rapports à la nature (Ferguson et Lovell, 2014). Face à l'ampleur de ces bouleversements et des contrastes, avec parfois de forts antagonismes entre modèles, l'identification de stratégies de développement pour les acteurs se trouve considérablement complexifiée. La recherche est interpellée pour apporter de nouveaux cadres de réflexion, dont un exemple est le concept de coexistence des modèles agricoles (Fournier et Touzard, 2014).

Pour enrichir ces nouveaux cadres de réflexions, le concept de vision du monde (worldview) connaît une nouvelle dynamique dans la littérature récente, et présente un potentiel important (Hedlund-de Witt, 2013). Ce concept a déjà été mobilisé en pratique pour diverses problématiques contemporaines, par exemple le changement climatique (Hulme, 2009; O'Brien et Hochachka, 2010), et le développement durable (Norgaard, 1988). Dans le domaine agricole, il a déjà permis de mieux comprendre certains verrous et leviers d'une transition vers des formes d'agriculture plus écologiques (Jordan et al., 2008; Sanford 2011; Hatt et al., 2016). Cependant, alors que l'utilité de ce concept est de plus en plus largement reconnue, il est rarement mobilisé dans le cadre d'une théorie scientifique formalisée et intégrative (Johnson et al., 2011). À ma connaissance, un tel cadre n'a pas même encore été testé dans le domaine de l'agriculture, afin de penser globalement le développement des systèmes. Dans cet article, une approche évolutive du concept est mobilisée, basée sur le cadre transdisciplinaire de la théorie intégrale (Wilber, 2000; Esbjörn-Hargens, 2010). Dans la première partie, je présente tout d'abord le concept de vision du monde en général et dans le cadre de cette théorie intégrale, et quatre grands types de vision du monde identifiés dans la littérature. Ensuite, je propose une déclinaison de ces quatre visions dans le domaine de l'agriculture, avant de montrer comment l'approche évolutive proposée constitue une nouvelle façon de penser le développement agricole, et quelles sont ses implications originales pour les acteurs.

\section{Une approche évolutive des «visions du monde "}

Le concept de vision du monde revêt des acceptions variées. En première approche, il peut se définir de façon générale comme un «système structurant et inéluctable de signification et de création de sens, qui informe comment les humains interprètent, promulguent et co-créent la réalité » (Hedlund-de Witt, 2013). Selon une autre définition de van Egmond et de Vries (2011), une vision du monde est une «combinaison des orientations d'une personne en termes de valeurs, et de sa compréhension du monde et des possibilités qu'il offre ». De nombreuses autres interprétations sont possibles, qui diffèrent notamment sur la nature des liens entre visions du monde et valeurs des individus (Naugle, 2002; Sire, 2015). À l'origine du concept, la notion de vision $\mathrm{du}$ monde a une longue et riche histoire en philosophie, avec les apports successifs notamment de Kant, Goethe, Hegel, Nietzsche, Heidegger et des postmodernistes (Derrida, Foucault...) (Hedlund-de Witt, 2013). Dans le domaine scientifique, la discipline sociologique s'est appropriée très tôt certains éléments de la notion (Weber). Aujourd'hui, des questions relatives aux visions du monde sont traitées dans des disciplines variées, incluant par exemple la sociologie pragmatique (Boltanski et Thévenot, 1991), la systémique (Bawden, 2006), la psychologie (Johnson et al., 2011) ou l'écologie (Beddoe et al., 2009). Par ailleurs, en plus de ses contributions particulières à différentes disciplines, le concept de vision du monde a aussi acquis une place centrale dans certaines théories transdisciplinaires, comme la théorie intégrale (Wilber, 2000). La théorie intégrale constitue une tentative d'intégrer autant d'approches, de théories et de penseurs que possible dans un cadre commun (EsbjörnHargens, 2010). Dans ce sens, elle a été présentée comme « une théorie du tout » visant à « rassembler des paradigmes séparés dans un réseau d'approches interconnectées qui s'enrichissent mutuellement» (Esbjörn-Hargens, 2010). Dans le cadre de cette théorie intégrale, le concept de vision du monde intègre à la fois le caractère construit (Kant) et historiquement et culturellement situé (Hegel) de nos positions, tout comme leurs liens avec les relations de pouvoir (Foucault) (Naugle, 2002; Sire, 2015). Cependant, la vision du monde n'est pas vue ici comme quelque chose qu'il faudrait mettre au jour pour mieux s'en affranchir: le caractère inévitable et utile de cadres structurants pour le fonctionnement de la pensée humaine est pleinement assumé, en intégrant les données des sciences cognitives et la psychologie (Wilber, 2000). Un apport particulièrement original de la théorie intégrale, émergeant 
du croisement de différentes approches, est de proposer une corrélation entre les évolutions collectives (socioculturelles) et le développement individuel (psychologique) des visions du monde (Wilber, 2000). Ainsi, dans la lignée théorique de l'épistémologie génétique de Jean Piaget, différentes visions $\mathrm{du}$ monde peuvent être vues comme des stades successifs dans le développement individuel et collectif.

Plusieurs méthodes permettent d'étudier les visions du monde, comme par exemple: des enquêtes sociales parmi les populations actuelles; des apports historiques et bibliographiques dans les domaines psychologiques et philosophiques; l'étude des cultures et des religions (van Egmond et de Vries, 2011). Sur la base de telles approches, plusieurs typologies de vision du monde ont été proposées, avec des nombres variables de types (jusqu'à huit selon Beck et Cowan, 2014). Dans cet article, pour rester le plus simple possible, nous nous baserons sur trois grands types de vision du monde: Traditionnelle; Moderne; Postmoderne (Hedlund-de Witt, 2013). Ces visions ont été clairement mises en évidence dans la littérature pour les civilisations occidentales contemporaines (Hedlund-de Witt, 2013), et sembleraient présenter certaines caractéristiques universelles (Wilber, 2000). Par ailleurs, en plus de ces trois visions du monde, nous considérerons une quatrième vision en émergence (Intégrale) suggérée par un nombre croissant de chercheurs (Wilber, 2000; van Egmond et de Vries, 2011; Beck et Cowan, 2014...). Une des caractéristiques de cette vision en émergence serait de lier la rationalité à la spiritualité, comme le propose d'ailleurs par exemple la théorie intégrale (Wilber, 2000). En France, cette vision émergente pourrait correspondre par exemple à la vision complexe transdisciplinaire initiée par Morin (2008), qui accorde une place très significative au mystère et à la spiritualité, et présente des consonances fortes avec la théorie intégrale (Gidley, 2013). Pour distinguer les quatre types de vision du monde, Hedlundde Witt (2013) s'appuie sur cinq dimensions :

- ontologique (postulats sur la nature de la réalité);

- épistémologique (postulats sur la connaissance de cette réalité) ;

- axiologique (valeurs portées par les individus);

- anthropologique (nature de l'être humain et sa place dans l'univers);

- sociétale (comment organiser la société).

Une description concise des quatre types avec une attention pour ces cinq dimensions est donnée dans le Tableau 1. Cette façon de décrire les visions du monde n'est évidemment pas la seule possible (voir par exemple O'Brien et Hochachka, 2010), mais elle me semble particulièrement structurante. Il est important de noter que ces quatre visions du monde peuvent se décliner d'une immense diversité de manières. Par exemple, une très grande diversité de religions correspond à un type de vision du monde Traditionnel (Wilber, 2000). De même, les visions Moderne, Postmoderne et Intégrale peuvent prendre une immense diversité de formes.

Selon l'approche évolutive des visions du monde proposée par la théorie intégrale, le développement des quatre visions est lié séquentiellement: la vision Traditionnelle «précède» la vision Moderne, qui «précède» la vision Postmoderne, qui elle-même «précède» la vision Intégrale, que ce soit chez l'individu ou dans les sociétés. En d'autres termes, on peut dire que la vision Intégrale «intègre et transcende» la vision
Postmoderne, qui elle-même à la fois « intègre et transcende » la vision Moderne, etc. (Wilber, 2000). Cependant, il est très important de souligner que la notion de développement n'est pas prise ici comme synonyme de progrès. Bien au contraire, Wilber (2000) insiste sur le fait que chacune des quatre visions du monde est «partiellement correcte», c'est-à-dire qu'elle a une valeur intrinsèque et peut contribuer de façon significative au fonctionnement d'un « tout» interconnecté plus large. De la même manière, aucune des quatre visions du monde n'est intrinsèquement «meilleure» qu'une autre. En revanche, chaque vision présente des formes d'expression plus ou moins favorables au fonctionnement de ce «tout» plus large. Par exemple, dans une optique de développement, la vision Traditionnelle peut être très efficace et positive dans l'urgence d'une crise humanitaire (charité). Cependant, si elle va jusqu'à instaurer des situations de dépendance humanitaire, elle peut devenir un obstacle très préjudiciable à la construction des capacités des populations locales sur le long terme (Brown, 2005). Par ailleurs, les quatre types de vision du monde ont tous le potentiel pour être écologiquement pertinents (EsbjörnHargens, 2010). Enfin, il est essentiel de bien comprendre que ces visions du monde ne correspondent aucunement à une caractérisation rigide des individus et des sociétés, et doivent être considérées avec un point de vue complexe. En particulier, chaque individu n'a pas simplement une seule et même vision du monde pour tout, mais plutôt une vision du monde prédominante (souvent), avec une immense diversité de déclinaisons possibles (Wilber, 2000).

Enfin, dans le cadre du présent article, il me semble très éclairant de bien remarquer que le concept de vision du monde lui-même prend une signification différente selon la vision du monde de celui qui l'utilise (Sire, 2015). Ainsi, à l'origine, la notion de vision du monde est typiquement Postmoderne, permettant de penser la connaissance comme «relative et contextualisée» (Tab. 1). En cela, cette notion se distingue clairement du positivisme (croyance en une réalité objective) caractéristique de la vision du monde Moderne. L'approche évolutive des visions du monde proposée dans cet article correspond quant à elle davantage à une vision du monde Intégrale, de par son niveau élevé de transdisciplinarité, qui intègre et transcende les approches antérieures multiples (van Egmond et de Vries, 2011).

\section{Application au monde agricole}

Une déclinaison des quatre visions du monde en quatre visions de l'agriculture est proposée dans le Tableau 2. Cette proposition correspond à une construction personnelle, ici esquissée à très grands traits. Cette esquisse subjective pourrait être critiquée, discutée et développée longuement pour rendre justice à la complexité des systèmes agricoles et alimentaires actuels (Hubert et al., 2013; Fournier et Touzard, 2014...) et du passé (Mazoyer et Roudart, 1997).

De façon synthétique, les visions Moderne et Postmoderne sont ici distinguées notamment par la mise en avant des notions de sécurité alimentaire pour la première, et de souveraineté pour la seconde. La vision Intégrale, encore très peu répandue, correspond à des formes d'agriculture intégrant des dimensions scientifiques et spirituelles (voir Tab. 2 pour explications). 
Tableau 1. Description succincte des quatre grands types de vision du monde dans les civilisations occidentales contemporaines, d'après Hedlund-de Witt (2013).

Table 1. A concise description of four major ideal-typical worldviews in the contemporary West, after Hedlund-de Witt (2013).

Vision du monde Traditionnelle

La métaphysique religieuse n'est pas distinguée de la science. Beaucoup d'importance est donnée aux écritures, aux doctrines et aux leaders religieux. Un Dieu transcendant est souvent vu comme séparé du monde terrestre, et l'homme est fondamentalement différent de la nature. La relation avec la nature est fréquemment comprise en termes de domination ou d'intendance. La famille et la communauté sont souvent essentielles, ainsi que des valeurs comme la sobriété, l'obéissance, la discipline, la solidarité, l'humilité et le respect de la tradition

\section{Vision du monde Moderne}

La rationalité et la pensée critique permettent de se libérer de l'autorité religieuse. La vision de la réalité tend à être matérialiste. La science constitue la source ultime (et souvent unique) de connaissance fiable permettant l'accès à une réalité objective. Cette «objectivation» de la réalité a généré un dualisme entre le sujet et l'objet. Elle a permis d'immenses progrès scientifiques, technologiques et économiques, ainsi qu'une instrumentalisation de la nature. La science et la technologie sont vues comme les voies centrales du progrès. L'homme autonome (self-made man) a une position centrale. Des valeurs individualistes et hédonistes sont généralement dominantes : liberté, indépendance, succès, performance, reconnaissance sociale, confort, fun

\section{Vision du monde Postmoderne}

De multiples perspectives sur la réalité sont reconnues comme valables, et la prétention de la science à l'exclusivité est critiquée. La connaissance est vue comme relative et contextualisée. Une attitude critique vis-à-vis de la société moderne est souvent observée, et l'émancipation de groupes exclus ou opprimés est une préoccupation centrale. Des exemples emblématiques sont les mouvements sociaux depuis les années 1960, promouvant la paix, le multiculturalisme, les droits homosexuels, l'environnement... Les valeurs importantes sont la diversité, la créativité, l'authenticité, l'imagination...

\section{Vision du monde Intégrale}

Une caractéristique essentielle est la réflexivité, qui permet de synthétiser des éléments des autres visions du monde, éventuellement considérés a priori comme contradictoires : science et spiritualité, logique et imagination, humanité et nature... Ces perspectives opposées sont comprises comme des éléments d'un tout plus large, à un niveau de réalité plus profond. Cette perspective peut conduire à un sens profond de connexion avec la nature. Des préoccupations universelles et existentielles sont de première importance (sens de la vie, prise de conscience de l'humanité...)

Chaque type de vision de l'agriculture correspond à une façon de penser le développement agricole (avec aussi de nombreuses déclinaisons au sein de chaque type). Selon une vision typiquement Moderne, ce développement est selon moi souvent présenté comme un processus graduel d'intensification de la production, comme le décrit notamment la théorie de Boserup (1965). Selon cette théorie, les transformations des systèmes et les innovations sont stimulées de façon essentielle par l'accroissement de la densité de population. Dans le cadre d'une vision de l'agriculture Postmoderne, d'autres théories sur le développement des systèmes agricoles, souvent d'inspiration marxiste, mettent davantage l'accent sur des facteurs sociaux comme les relations de pouvoir (Sevilla Guzmán et Woodgate, 2013). Enfin, l'approche évolutive des visions du monde proposée dans cet article, correspondant à une vision Intégrale, permet de penser le développement des systèmes agricoles autrement qu'avec les visions majoritaires actuelles Moderne ou Postmoderne. En effet, selon cette perspective, les évolutions techniques et organisationnelles des systèmes peuvent être interprétées en lien avec les visions du monde des individus et des sociétés. Ainsi, avec l'avènement et la diffusion de la science agronomique, les visions du monde des acteurs de l'agriculture ont évolué d'une vision Traditionnelle dominante à une vision Moderne dominante. Dans les dernières décennies, on commence à constater la montée en puissance d'une vision Postmoderne, et l'émergence d'une vision Intégrale, qui pourrait prendre de l'importance à l'avenir. Ces évolutions des visions du monde ont des effets sur les composantes techniques et organisationnelles des systèmes, et réciproquement. Par exemple, la vision Moderne entraîne une « modernisation » concrète des systèmes (rationalisation de la fertilisation, de l'alimentation du bétail, du travail; machinisme, nouvelles technologies de l'information et de la communication...). Cette modernisation influe en retour sur les visions du monde des acteurs (ouverture sur le monde, prise de conscience des problèmes environnementaux...), qui influent en retour sur les systèmes techniques... Il n'y a évidemment rien d'automatique ni de linéaire dans cette coévolution des visions de l'agriculture. En particulier, la dimension politique est cruciale dans le sens donné au développement agricole et rural, et peut tout à fait rendre possible un passage «en sens inverse», d'une vision Moderne à une vision Traditionnelle par exemple.

En plus de ces considérations théoriques, l'approche proposée a aussi des implications pratiques concrètes pour les acteurs impliqués dans le développement agricole. En particulier, elle invite chacun à développer une réflexivité sur sa propre vision, et à mieux prendre en compte celle des autres. Ainsi, les chercheurs, ingénieurs ou politiques peuvent adapter leurs stratégies de développement aux différentes visions du monde des acteurs concernés par leurs projets. Cette approche me semble aller plus loin que certains travaux sur la coexistence, qui ont déjà montré l'intérêt d'une grande diversité de modèles agroalimentaires (associés à différentes visions du monde) dans un objectif de sécurité 
Tableau 2. Description succincte de quatre grands types de vision de l'agriculture dans les civilisations occidentales contemporaines (construction personnelle de l'auteur).

Table 2. A concise description of four major ideal-typical views of agriculture in the contemporary West (personal proposition of the author).

Vision de l'agriculture Traditionnelle

Cette vision correspond selon moi à la paysannerie avant la modernisation, encore très présente dans le monde et telle que l'a notamment décrite Mendras (1970). L'agriculture est une activité vivrière et peu marchande. Le paysan est soucieux de la reproduction de la cellule familiale, la solidarité est essentielle et le travail est une valeur suprême (Mendras, 1970). La religion a un rôle essentiel dans la compréhension du monde, avec des répercussions importantes sur les pratiques agricoles (interprétation des aléas...)

Vision de l'agriculture Moderne

Cette vision est indissociable de la science agronomique classique, et leurs évolutions sont étroitement liées. Aujourd'hui, le développement agricole selon cette vision est typiquement cadré par l'objectif de sécurité alimentaire et de développement durable, ou «comment nourrir la planète en 2050» (Inra, 2010; CGIAR, 2015). Cette déclinaison de la vision du monde Moderne en agriculture a considérablement évolué au cours du temps. À l'origine de l'application de la science agronomique à l'agriculture, les gains de production ont été mis en avant, puis les performances économiques. Aujourd'hui, la vision Moderne traduit typiquement les enjeux de développement durable par une injonction à l'intensification écologique, avec des concepts centraux comme l'efficience («produire plus avec moins ») et la multi-performance (économique, environnementale, sociale) (Inra, 2010). La notion de sécurité alimentaire se centre sur la dimension matérielle de l'agriculture et de l'alimentation (fourniture en calories et protéines), cette dimension pouvant être abordée de façon objective (de même que les «impacts environnementaux»). Enfin, la vision Moderne place de grands espoirs dans les nouvelles technologies (agriculture de précision, agriculture connectée, biologie prédictive...)

Vision de l'agriculture Postmoderne

Cette vision met en avant la notion de souveraineté alimentaire, qui à la fois «intègre et transcende» celle de sécurité alimentaire.

La souveraineté est définie comme «le droit de chaque nation de maintenir et d'élaborer sa propre capacité de produire ses propres aliments de base dans le respect de la diversité productive et culturelle» (Sommet mondial de l'alimentation, 1996). Dans ces termes, cette notion intègre la diversité des contextes territoriaux et leur dimension subjective. En conséquence, les systèmes agricoles plébiscités sont «situés » dans leurs sociétés et leurs territoires (appellations d'origine, produits de terroir, commerce équitable, agroécologie au sens d'Altieri (2009)...). L'agriculture industrielle est souvent vivement critiquée. La nature est considérée comme un «partenaire» avec lequel il faut composer (notion de services écosystémiques...). La recherche-développement est typiquement interdisciplinaire et participative, pour intégrer les composantes sociales du développement et des valeurs jugées essentielles comme la créativité. Des exemples de travaux emblématiques sont par exemple la caractérisation des farming styles (van der Ploeg, 2009), ou plus généralement la plupart des recherches soft system pour l'action (Darnhofer et al., 2012)

Vision de l'agriculture Intégrale

Cette vision est encore très peu répandue en agriculture. Elle insiste sur les synergies entre dimensions spirituelles et scientifiques du développement (un exemple médiatique est sans doute Pierre Rabhi, 2009). La subjectivité des animaux, voire des plantes, est considérée, conduisant à un sentiment profond de connexion avec la nature. Des exemples illustratifs récents dans la recherche académique sont peutêtre Buchanan (2013) et Cox (2014), qui adoptent un niveau très profond de réflexivité, avec une démarche résolument transdisciplinaire. Certains grands auteurs alternatifs peuvent probablement être également reconsidérés comme précurseurs dans cette optique (Steiner,

Fukuoka...)

alimentaire (Fournier et Touzard, 2014). En effet, dans ces travaux, la question des synergies entre systèmes est «subordonnée» à un objectif particulier, relevant en l'occurrence d'une vision Moderne (la sécurité alimentaire). En reconnaissant très explicitement la légitimité de différentes visions du monde, l'approche proposée ici invite profondément à se mettre « à la place » des acteurs et à développer de l'empathie. Les stratégies «gagnant-gagnant» peuvent alors reposer sur des «palettes de valeurs». Ainsi, par exemple, Schösler et Hedlund-de Witt (2012) ont montré que les mouvements biologiques et slow food pouvaient respecter et satisfaire en synergie à la fois des valeurs Traditionnelles (agricultures familiales, « recettes de grandmères »...), des valeurs Modernes (performances économiques, qualités organoleptiques, santé, innovation...), et des valeurs Postmodernes (bien-être animal, alimentation naturelle...). Par ailleurs, l'approche évolutive adoptée dans cet article rend explicite le fait que les visions du monde peuvent évoluer (au sein d'un type) et se transformer (d'un type à l'autre). Ces évolutions doivent s'envisager à différentes échelles de temps et d'espace. À l'échelle des individus et à court terme (temps d'un projet de recherche), les visions $\mathrm{du}$ monde changent rarement, et les conditions de ce changement sont mal connues (Brown, 2005). Pour cette raison, Hedlund-de Witt (2013) recommande généralement de valoriser des formes d'expression "positives » des visions du monde des acteurs, sans chercher à les transformer. Ceci revient à reconnaître que les individus « ont le droit d'être où ils sont» en termes de leur vision du monde dominante, et qu'ils doivent être respectés et honorés en tant que tels (Hedlund-de Witt, 2013). À plus long terme, les implications pourraient être encore plus considérables. En effet, chercher à comprendre le point de vue d'autrui (plutôt que le changer) amène à mettre «entre parenthèses » sa propre position et à s'ouvrir à la possibilité d'être changé soi-même. Selon Brown (2005), c'est justement cette ouverture qui permet d'obtenir des solutions vraiment participatives, émergentes et mutuellement transformatives. 


\section{Conclusion}

Une approche évolutive des visions du monde donne un cadre original et stimulant pour penser différemment l'évolution des systèmes agricoles. Elle invite aussi à développer une réflexivité sur sa propre vision du monde, à mieux prendre en compte celle des autres, et ainsi à s'engager dans des dynamiques de transformations mutuelles. De façon essentielle, cette approche ne doit pas être prise comme un modèle rigide pour classifier ou catégoriser des acteurs ou des organisations, ni pour prédire des évolutions futures. Le développement agricole est assurément une question extrêmement complexe, qui ne se laisse enfermer dans aucun cadre. Dans ce sens, l'approche proposée doit être considérée comme une perspective particulière, parmi beaucoup d'autres approches possibles (historiques, socioéconomiques, politiques, institutionnelles...). Par ailleurs, nous avons vu que le concept de vision du monde prend une signification différente selon la vision du monde de celui qui l'utilise. En particulier, l'approche évolutive du concept trouvera sans doute davantage de résonance dans le cadre d'une vision du monde Intégrale. Cependant, même sans nécessairement adhérer complètement à l'ensemble de l'approche et à ses implications radicales, chacun peut y trouver une source d'inspiration pour aborder la coexistence des systèmes de façon plus constructive, à la fois bienveillante et engagée.

\section{Références}

Altieri MA. 2009. Agroecology, small farms, and food sovereignty. Monthly Review 61(3): 102.

Bawden R. 2006. A systemic evaluation of an agricultural development: a focus on the worldview challenge. Systems concepts in evaluation. Point Reys, CA: Edge Press of Inverness, pp. 35-46.

Beck DE, Cowan C. 2014. Spiral dynamics: mastering values, leadership and change. John Wiley \& Sons.

Beddoe R, Costanza R, Farley J, Garza E, Kent J, Kubiszewski I, et al. 2009. Overcoming systemic roadblocks to sustainability: the evolutionary redesign of worldviews, institutions, and technologies. Proceedings of the National Academy of Sciences 106(8): 2483-2489.

Boltanski L, Thévenot L. 1991. De la justification. Les économies de la grandeur. Paris : Gallimard.

Boserup E. 1965. The conditions of agricultural growth. The economics of agriculture under population pressure. London and New York: George Allen \& Unwin Ltd, 124 p.

Buchanan J. 2013. An integral approach to agroecological research. Doctoral dissertation, University of Wisconsin (Madison).

Brown B. 2005. Theory and practice of integral sustainable development. AQAL Journal of Integral Theory and Practice 1(2): 2-39.

CGIAR Consortium. 2015. CGIAR strategy and results framework 2016-2030: overview.

Conway G. 1999. The doubly green revolution: food for all in the twenty-first century. Ithaca, NY: Cornell University Press.

Cox TE. 2014. Integral agriculture: taking seriously the mindset of the farmer, the interiority of the beings on the farm, and a metaphysics that connects them. Doctoral dissertation, Iowa State University.

Darnhofer I, Gibbon D, Dedieu B. 2012. Farming systems research: an approach to inquiry. In: Farming systems research into the $21 \mathrm{st}$ century: the new dynamic. Netherlands: Springer, pp. 3-31.
Esbjörn-Hargens S. 2010. An overview of integral theory: an allinclusive framework for the 21 st century (resource paper No. 1). Boulder, CO: Integral Institute.

Ferguson RS, Lovell ST. 2014. Permaculture for agroecology: design, movement, practice, and worldview. A review. Agronomy for Sustainable Development 34(2): 251-274.

Fournier S, Touzard JM. 2014. La complexité des systèmes alimentaires: un atout pour la sécurité alimentaire? VertigO la revue électronique en sciences de l'environnement 14(1).

Gidley JM. 2013. Global knowledge futures: articulating the emergence of a new meta-level field. Integral Review 9(2): 145-172.

Hatt S, Artru S, Brédart D, Lassois L, Francis F, Haubruge E, et al. 2016. Towards sustainable food systems: the concept of agroecology and how it questions current research practices. A review. Biotechnology, Agronomy, Society and Environment 20(S1): 215-224.

Hedlund-de Witt A. 2013. Worldviews and the transformation to sustainable societies: an exploration of the cultural and psychological dimensions of our global environmental challenges. Doctoral dissertation, Vrieje University (Amsterdam, the Netherlands).

Hubert B, Goulet F, Magnani S, Tallon H, Huguenin J. 2013. Agriculture, modèles productifs et options technologiques: orientations et débats. Natures Sciences Sociétés 21(1): 71-76.

Hulme M. 2009. Why we disagree about climate change: understanding controversy, inaction and opportunity. Cambridge: Cambridge University Press.

Inra. 2010. Document d'orientation. Inra 2010-2020. Une science pour l'impact.

Issac H, Poyat M. 2015. Les défis de l'agriculture numérique connectée dans une société numérique. 16 propositions pour repenser la production, la distribution et la consommation alimentaires à l'ère du numérique. Reconnaissance numérique, $103 \mathrm{p}$.

Johnson KA, Hill ED, Cohen AB. 2011. Integrating the study of culture and religion: toward a psychology of worldview. Social and Personality Psychology Compass 5(3): 137-152.

Jordan NR, Bawden RJ, Bergmann L. 2008. Pedagogy for addressing the worldview challenge in sustainable development of agriculture. Journal of Natural Resources and Life Sciences Education 37: 92-99

Mazoyer M, Roudart L. 1997. Histoire des agricultures du monde : du néolithique à la crise contemporaine. Paris : Éditions du Seuil, 545 p.

McIntyre BD. 2009. International assessment of agricultural knowledge, science and technology for development (IAASTD): global report.

Mendras H. 1970. La fin des paysans : changement et innovations dans les sociétés rurales françaises. Colin, vol. 110.

Morin E. 2008. La méthode. Paris : Seuil, coll. « Opus », 2 volumes.

Naugle DK. 2002. Worldview: the history of a concept. UK: W.B. Eerdmans Publishing.

Norgaard RB. 1988. Sustainable development: a co-evolutionary view. Futures 20(6): 606-620.

O'Brien K, Hochachka G. 2010. Integral adaptation to climate change. Journal of Integral Theory and Practice 5(1): 89-102.

Rabhi P. 2009. Vers la sobriété heureuse. Arles : Actes Sud, 144 p.

Sanford AW. 2011. Ethics, narrative, and agriculture: transforming agricultural practice through ecological imagination. Journal of Agricultural and Environmental Ethics 24: 283-303. doi:10.1007/ s10806-010-9246-6.

Schösler H, Hedlund-de Witt A. 2012. Sustainable protein consumption and cultural innovation. What businesses, organizations, and governments can learn from sustainable food trends in Europe and the United States. Amsterdam: Reprografie. 
Sevilla Guzmán E, Woodgate G. 2013. Agroecology: foundations in agrarian social thought and sociological theory. Agroecology and Sustainable Food Systems 37(1): 32-44.

Sire JW. 2015. Naming the elephant: worldview as a concept. USA: InterVarsity Press.

Tilman D, Balzer C, Hill J, Befort BL. 2011. Global food demand and the sustainable intensification of agriculture. Proceedings of the National Academy of Sciences 108(50): 20260-20264. van Egmond ND, de Vries HJM. 2011. Sustainability: the search for the integral worldview. Futures 43(8): 853-867.

van der Ploeg JD. 2009. The new peasantries: struggles for autonomy and sustainability in an era of empire and globalization. UK: Routledge.

Wilber K. 2000. Sex, ecology, spirituality. Colorado: Shambhala Publications.

Wilkinson J. 2009. The globalization of agribusiness and developing world food systems. Monthly Review 61(4): 38.

Citation de l'article : Rigolot C. 2017. Une approche évolutive des « visions du monde» pour penser les transformations de l'agriculture. Cah. Agric. 26: 36001. 